\section{Anatomic location and route of extension of the trigeminal nerve schwannomas}

\section{Sir,}

Chowdhury et al..$^{[1]}$ in their retrospective study highlighted the complex developmental pattern of the trigeminal nerve (TN) schwannomas and Ghosh et al..$^{[2]}$ in their interesting case report pointed out their extracranial extension into the orbit. The current letter focuses on the value of knowledge of the exact anatomic location and route of extension of these rare benign intracranial tumors.

The TN schwannomas most commonly arise from the gasserian ganglion within the Meckel cave [Figures 2 and $1 \mathrm{~b}$ ] but may also originate more proximally from the nerve root or more distally from one of the three post-ganglionic TN branches [Figures $1 \mathrm{c}$ and $\mathrm{f}$ ]. The ophthalmic nerve $\left(\mathrm{V}_{1}\right)$ is more commonly affected compared to the maxillary $\left(\mathrm{V}_{2}\right)$ or the mandibular nerve $\left(\mathrm{V}_{3}\right)$. The small parasympathetic fibers from the sphenopalatine ganglion may be an alternative pathway of the autonomic system for the tumor extension. ${ }^{[3]}$ Schwannomas of the motor division are very rare and their existence is correlated with neurofibromatosis. ${ }^{[1]}$

Schwannomas of the TN may predominantly be located in a single cranial fossa (middle or posterior) or may be extended in multiple compartments of the skull. Their development and extracranial expansion occupy several pathways, such as the subdural (cerebellopontine angle) and the intradural space (lateral cavernous sinus wall and Meckel cave), the epidural area, the orbit (through optic canal and superior orbital fissure), the pterygopalatine (through foramen rotundum) and the infratemporal fossa (through foramen ovale) [Figure 1e], the nasal cavity, the maxillary sinus ${ }^{[4]}$ and rarely the deep parapharyngeal space. ${ }^{[5]}$ Occasionally, schwannomas reach enormous dimensions resulting in complex clinical manifestations.

Clinical features vary according to the anatomical site of origin, the direction and extent of tumor growth. Numbness, paresthesia or hypoesthesia in one or more

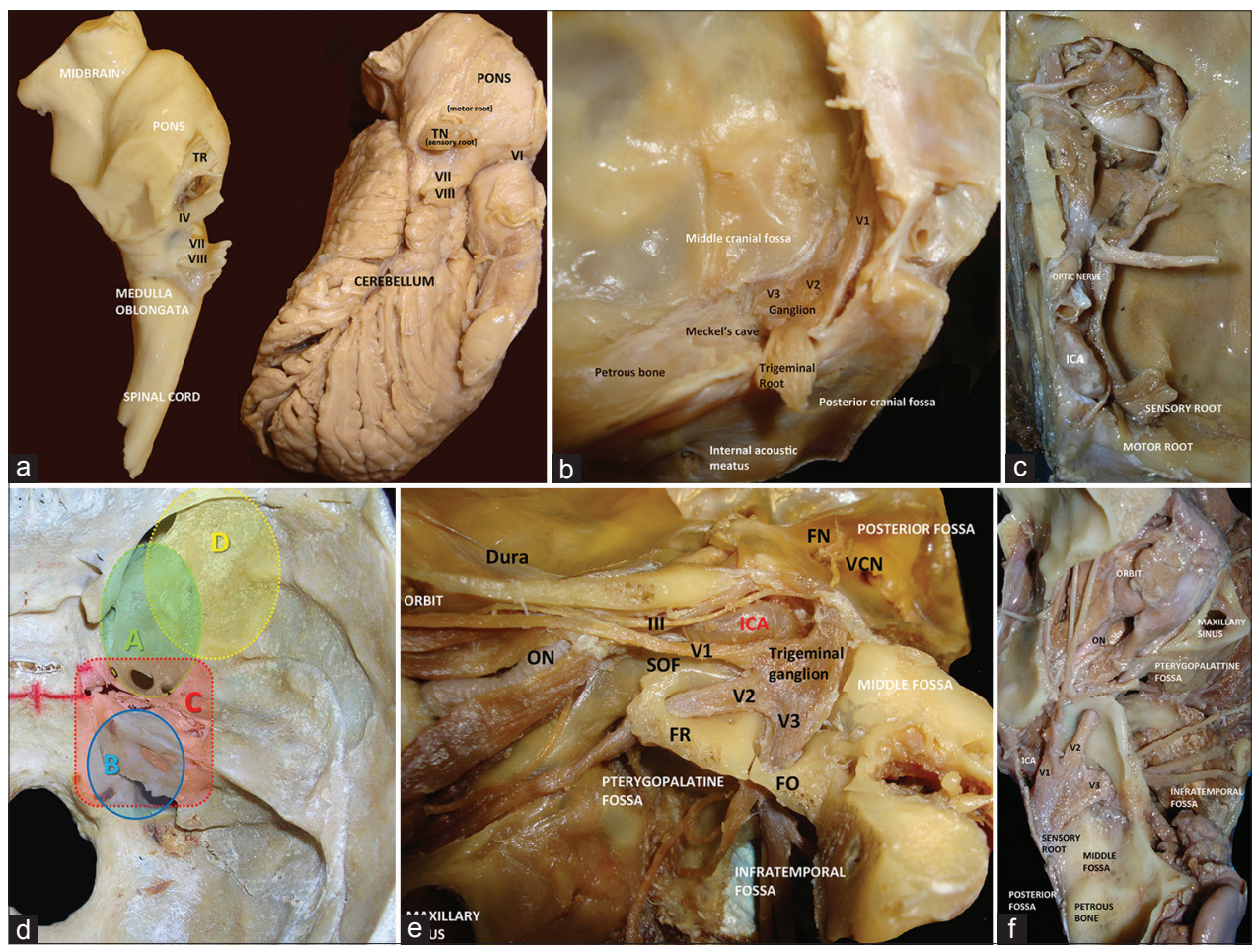

Figure 1: $(a$ and b) A large sensory root (TR) enters the brainstem at the pons. The adjacent cranial nerves are also depicted in a lateral right view of a formalin fixed specimen, IV-trochlear nerve, VI-abducens nerve, VII-facial nerve and VIII-vestibulocochlear nerve. (c) Immediately adjacent to the sensory root, a smaller motor root. (d) Sites of location of the trigeminal nerve (TN) schwannomas and their extension, A (green) - middle cranial fossa, B (blue)—cerebellopontine angle, C (red)—middle and posterior cranial fossae, D (yellow) —extracranial extension. (e and f) The three divisions of TN: The ophthalmic nerve (V1) (superior orbital fissure-SOF), the maxillary nerve (V2) (foramen rotundum-FR) and the mandibular nerve (V3) (foramen ovale-FO) converge on the trigeminal (gasserian) ganglion located within Meckel cave. ON $=$ optic nerve, $\mathrm{FN}=$ facial nerve, $\mathrm{VCN}=$ vestibulocochlear nerve, III = occulomotor nerve, ICA = internal carotid artery 


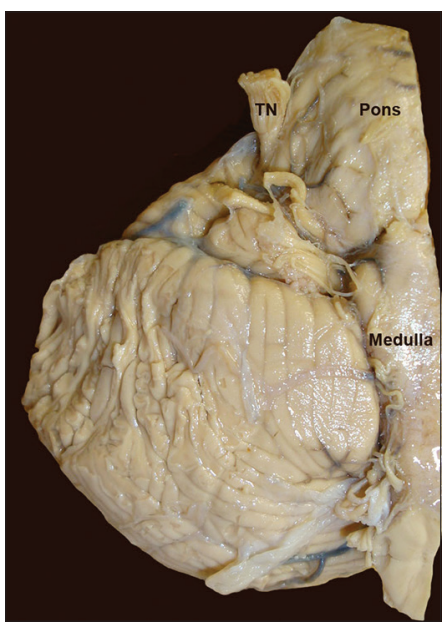

Figure 2: The emersion of the trigeminal nerve (TN) at the pons (right anterior aspect)

branches is frequently encountered, while anesthesia of the entire nerve raises the suspicion of malignancy. Typical neuralgic pain or atypical facial pain ${ }^{[6]}$ and a decreased or absent corneal reflex are also reported. The constant pain is associated with middle cranial fossa lesions due to the relative fixed location of the ganglion in the trigeminal impression of petrous bone, while intermittent pain is associated with posterior fossa tumors due to the compression of the relatively mobile TN roots. Moreover, the decreased motor function appeared as weakness of the masseter, temporalis and pterygoid muscles. In sporadic cases, the TN schwannomas may present with sudden onset of headache due to intratumoral or subarachnoid hemorrhage. Their extension into the cavernous sinus may result in diplopia, and further expansion into the orbital apex may cause exophthalmos and visual disturbances. The direct compression or traction on geniculate ganglion and vestibulocochlear nerve may cause facial nerve dysfunction and hearing loss, respectively. Focal seizures, cerebellar and lower cranial nerve signs, pathological laughter associated with compression of the medial part of the temporal lobe or the brainstem and signs of increased intracranial pressure are also mentioned. ${ }^{[4]}$

Various skull base approaches and microsurgical techniques were planned for the complete resection of the tumors depending on their size and location [Figure 1d] with the aim to preserve the TN function. Neurosurgeons select the appropriate approach based on their ability. ${ }^{[1]}$ Patients with recurrent tumors and previously irradiated due to the existent fibrosis are treated leaving a small residual tumor attached to the brainstem and the surrounding vital structures due to the risk of traction injury and perforation damage ${ }^{[7]}$ Recent advances in neuroimaging allow the exact visualization of the tumor size, location and extension pattern. The bone infiltration, the erosion of adjacent bony structures, the enlargement of foramina and the atrophy of the ipsilateral masseter and pterygoid muscles are well demonstrated on computed tomography. ${ }^{[8]}$ Thus, we estimate that the meticulous knowledge of the anatomy of the TN area is crucial for the early diagnosis of the lesion to prevent instances of morbidity and mortality and plan the total surgical resection minimizing brain exposure and avoiding surgical complications.

Konstantinos Natsis, Maria Piagkou ${ }^{1}$

Professor and Director of the Department Anatomy, School of Medicine, Aristotle University of Thessaloniki, Thessaloniki, ${ }^{1}$ Department of Anatomy, School of Medicine, National and Kapodistrian University of Athens, Athens, Greece

Address for correspondence: Dr. Maria Piagkou, Department of Anatomy, School of Medicine, National and Kapodistrian University of Athens, Mikras Asias 75, Athens - 11527, Greece. E-mail: mapian@med.uoa.gr

\section{References}

1. Chowdhury FH, Haque MR, Kawsar KA, Sarker MH, Hasan M, Goel AH. Intracranial nonvestibular neurinomas: Young neurosurgeons' experience. J Neurosci Rural Pract 2014; 5:231-43.

2. Ghosh S, Das D, Varshney R, Nandy S. Orbital extension of trigeminal schwannoma. J Neurosci Rural Pract 2015;6:102-4.

3. Chacko AG, Natarajan MS, Prabhu K, Chacko G. Schwannoma of supraorbital nerve presenting as a subfrontal tumor. Neurol India 2014; 62:96-8.

4. Yoshida K, Kawase T. Trigeminal neurinomas extending into multiple fossae: Surgical methods and review of the literature. J Neurosurg 1999;91:202-11.

5. Jimbo H, Kamata S, Miura K, Asamoto S, Masubuchi T, Ikeda Y, et al. Surgical management of giant trigeminal schwannomas extending into the parapharyngeal space. Acta Neurochir (Wien) 2009; 151:335-40.

6. Piagkou MN, Skandalakis P, Piagkos G, Demesticha T. Trigeminal pain and its distribution in different trigeminal nerve branches. Anesth Pain Med 2012;1:271-2.

7. Behari S, Tyagi I, Banerji D, Kumar V, Jaiswal AK, Phadke RV, et al. Postauricular, transpetrous, presigmoid approach for extensive skull base tumors in the petroclival region: The successes and the travails. Acta Neurochir (Wien) 2010; 152:1633-45.

8. MacNally SP, Rutherford SA, Ramsden RT, Evans DG, King AT. Trigeminal schwannomas. Br J Neurosurg 2008;22:729-38.

\begin{tabular}{|l|l|}
\hline \multicolumn{2}{|c|}{ Access this article online } \\
\hline Quick Response Code: & Website: \\
\hline & www.ruralneuropractice.com \\
\cline { 2 - 2 } & \\
\hline
\end{tabular}

